\title{
Some integrals of hypergeometric functions
}

\author{
by András BIRÓ
}

\section{A. Rényi Institute of Mathematics, Hungarian Academyof Sciences} 1053 Budapest, Reáltanoda u. 13-15., Hungary; e-mail: biroand@renyi.hu

\begin{abstract}
We consider a certain definite integral involving the product of two classical hypergeometric functions having complicated arguments. We show in this paper the surprising fact that this integral does not depend on the parameters of the hypergeometric functions.
\end{abstract}

\section{Statement of the results}

1.1. Our aim in this paper is to prove Theorem 1.1 stated below.

We write $F\left(\begin{array}{c}\alpha, \beta \\ \gamma\end{array} ; z\right)$ for the Gauss hypergeometric function (instead of the notation $F(\alpha, \beta, \gamma ; z)$ used in $[3])$.

THEOREM 1.1. Let $0<T<S<1$, and let $t$ be any complex number. Then

$$
A_{t}(S, T):=\int_{T}^{S} \frac{F\left(\begin{array}{c}
2 i t,-2 i t \\
\frac{1}{2}
\end{array} \frac{(1+\sqrt{z})(\sqrt{z}-\sqrt{T})}{2(1-\sqrt{T}) \sqrt{z}}\right) F\left(\begin{array}{c}
i t,-i t \\
\frac{1}{2}
\end{array} ;-\frac{(S-z)(1-z)}{(1-\sqrt{S})^{2} z}\right)}{(1-z) \sqrt{z-T} \sqrt{S-z}} d z
$$

equals

$$
\frac{\pi}{\sqrt{1-T} \sqrt{1-S}}
$$

Research partially supported by the NKFIH (National Research, Development and Innovation Office) Grants No. K104183, K109789, K119528, ERC_HU_15 118946, and ERCAdG Grant no. 321104

2000 Mathematics Subject Classification: 33C05, 33C60

Key words and phrases: hypergeometric functions, integral formulas 
1.2. We note that the numerator in (1.1) is symmetric in $S$ and $T$. Indeed, by applying the quadratic transformation

$$
F\left(\begin{array}{c}
i t,-i t \\
\frac{1}{2}
\end{array} 4 w(1-w)\right)=F\left(\begin{array}{c}
2 i t,-2 i t \\
\frac{1}{2}
\end{array} ;\right) \text { for }-\infty<w \leq \frac{1}{2}
$$

(this follows from [2], p 999, 9.133) for the second hypergeometric function one has

$$
F\left(i t,-i t ;-\frac{(S-z)(1-z)}{(1-\sqrt{S})^{2} z}\right)=F\left(2 i t,-2 i t ; \frac{(1+\sqrt{z})(\sqrt{z}-\sqrt{S})}{2(1-\sqrt{S}) \sqrt{z}}\right)
$$

We have decided to use the left-hand side of (1.3) in Theorem 1.1 because the argument of the hypergeometric function there is a bit simpler than on the right-hand side.

We also note that we could not simply apply (1.2) for the first hypergeometric function in (1.1) because the argument of the function there can be any number between 0 and 1 , and we cannot apply (1.2) for $w>\frac{1}{2}$.

1.3. We think that the identity of Theorem 1.1 is interesting in its own right, but we mention that we observed it while studying the integral operator

$$
g(S)=\int_{0}^{S} \frac{F\left(\begin{array}{c}
i t,-i t \\
\frac{1}{2}
\end{array} ;-\frac{(S-z)(1-z)}{(1-\sqrt{S})^{2} z}\right)}{\sqrt{S-z}} f(z) d z
$$

where $f$ and $g$ are functions on $(0,1)$. In fact, one can give the inverse of this transform using Theorem 1.1, we intend to show it in a forthcoming paper.

We also mention that when $T$ and $S$ are fixed, but $t$ is a real number and tends to infinity, then the integrand in the integral defining $A_{t}(S, T)$ may be exponentially large, so it is an interesting fact that $A_{t}(S, T)$ itself is bounded (which follows, of course, from Theorem $1.1)$.

\section{Preliminary lemmas}

We first need a lemma which shows that $F\left(\begin{array}{c}-i t, i t \\ \frac{1}{2}\end{array} ;-x\right)$ is in fact a trigonometric function, and using this fact we also show a product formula for this function.

For a complex number $z \neq 0$ we set its argument in $(-\pi, \pi]$, and write $\log z=\log |z|+$ $i \arg z$, where $\log |z|$ is real. We define the power $z^{s}$ for any $s \in \mathbf{C}$ by $z^{s}=e^{s \log z}$. 
LEMMA 2.1. (i) For every complex $t$ and for every real number $x>-1$ we have that

$$
F\left(\begin{array}{c}
-i t, i t \\
\frac{1}{2}
\end{array}-x\right)=\frac{1}{2}\left((\sqrt{x+1}+\sqrt{x})^{2 i t}+(\sqrt{x+1}+\sqrt{x})^{-2 i t}\right)
$$

and one can also write it as

$$
F\left(\begin{array}{c}
-i t, i t \\
\frac{1}{2}
\end{array} ;-x\right)=\cos (2 t \log (\sqrt{x+1}+\sqrt{x}))=\cos (2 t \log (\sqrt{x+1}-\sqrt{x})) .
$$

(ii) For every complex $t$ and for every real numbers $x>0$ and $y>0$ we have that the product

$$
2 F\left(\begin{array}{c}
-i t, i t \\
\frac{1}{2}
\end{array} ;-x\right) F\left(\begin{array}{c}
-i t, i t \\
\frac{1}{2}
\end{array} ;-y\right)
$$

equals

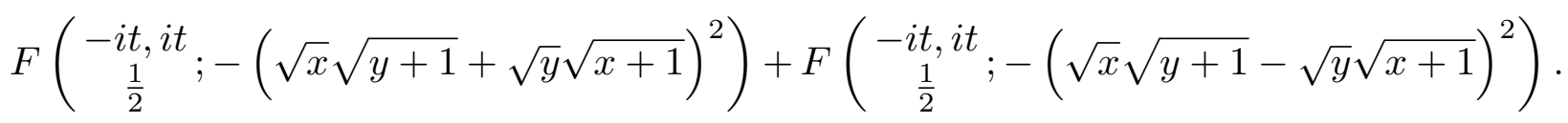

Proof. For the case $x>0$ formula (2.1) follows from [2], p. 998, 9.131.1 and [3], (1.5.19).

Then it follows by analytic continuation also for $x>-1$, taking into account that

$$
(\sqrt{x+1}+\sqrt{x})^{-2 i t}=(\sqrt{x+1}-\sqrt{x})^{2 i t}
$$

Then (2.2) follows at once, the second equality there follows by the evenness of the cosine function.

Now, writing

$$
X=(\sqrt{x} \sqrt{y+1}+\epsilon \sqrt{y} \sqrt{x+1})^{2}
$$

we have

$$
X+1=(\sqrt{x+1} \sqrt{y+1}+\epsilon \sqrt{x} \sqrt{y})^{2}
$$

for $\epsilon=1$ and also for $\epsilon=-1$, hence

$$
F\left(\begin{array}{c}
-i t, i t \\
\frac{1}{2}
\end{array} ;-X\right)=\cos (2 t \log ((\sqrt{x+1}+\sqrt{x})(\sqrt{y+1}+\epsilon \sqrt{y})))
$$

Part (ii) then follows by the trigonometric identity [2], p 29, 1.314.3. The lemma is proved. 
The following three lemmas are easy consequences of Lemma 2.1 and the identity

$$
\frac{1}{2 \pi} \int_{0}^{\infty} \frac{\Gamma(a \pm i s) \Gamma(b \pm i s) \Gamma(c \pm i s)}{\Gamma( \pm 2 i s)} d s=\Gamma(a+b) \Gamma(a+c) \Gamma(b+c),
$$

which is valid for $a \geq 0$ and $b, c>0$. This is formula (3.6.1) of [1] for positive numbers $a, b$ and $c$, but by taking $a \rightarrow 0+0$ the same formula holds for $a=0$ and positive $b$ and $c$.

LEMMA 2.2. For every $A>-1$ and for every $T \geq 0$ we have that

$$
\frac{1}{2 \pi} \int_{0}^{\infty} \frac{\Gamma( \pm i s) \Gamma\left(\frac{1}{2} \pm i s\right) \Gamma\left(\frac{1}{2}+T \pm i s\right)}{\Gamma( \pm 2 i s)} F\left(\begin{array}{c} 
\pm i s \\
\frac{1}{2}
\end{array} ;-A\right) d s
$$

equals

$$
\Gamma\left(\frac{1}{2}\right) \Gamma(1+T) \Gamma\left(\frac{1}{2}+T\right)(1+A)^{-\frac{1}{2}-T} .
$$

Proof. Using Lemma 2.1 (i) and the Stirling formula to give an upper bound it is trivial by analytic continuation that it is enough to prove the statement for $0<A<1$.

Using (2.5) for $0<A<1$ we have that (2.6) equals

$$
\sum_{k=0}^{\infty} \frac{(-A)^{k}}{k !\left(\frac{1}{2}\right)_{k}} \Gamma\left(\frac{1}{2}+k\right) \Gamma(1+T) \Gamma\left(\frac{1}{2}+k+T\right),
$$

and this equals (2.7) by the binomial theorem. The lemma is proved.

LEMMA 2.3. For every $A>-1$ and for every $r>0$ we have that

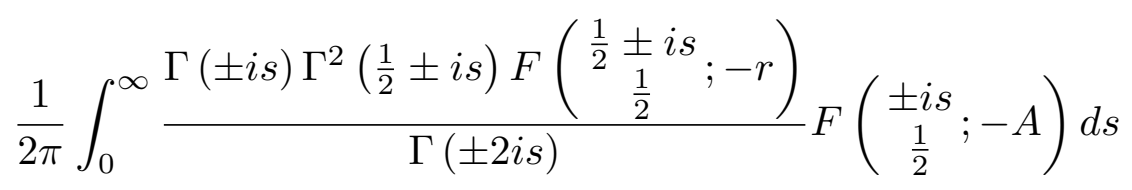

equals

$$
\pi(1+A)^{\frac{1}{2}} \frac{1}{1+r+A}
$$

Proof. Note first that

$$
F\left(\frac{1}{2}^{\frac{1}{2}} ; i s\right)=(1+r)^{-\frac{1}{2}} \cos (2 s \log (\sqrt{r+1}+\sqrt{r}))
$$

by the third line of [2], p 999, 9.131.1 and by (2.2) above. By this formula and by (2.2) one sees by analytic continuation that it is enough to show the statement for fixed $A>0$ and for small enough positive $r$. In this case, using Lemma 2.2 we see that (2.8) equals

$$
\sum_{T=0}^{\infty} \frac{(-r)^{T}}{T !\left(\frac{1}{2}\right)_{T}} \Gamma\left(\frac{1}{2}\right) \Gamma(1+T) \Gamma\left(\frac{1}{2}+T\right)(1+A)^{-\frac{1}{2}-T} .
$$


By summing this geometric series the lemma follows.

LEMMA 2.4. For every $A>-1$ and for every $r>0$ and $B \geq 0$ we have that

$$
\frac{1}{2 \pi} \int_{0}^{\infty} \frac{\Gamma( \pm i s) \Gamma^{2}\left(\frac{1}{2} \pm i s\right) F\left(\begin{array}{c}
\frac{1}{2} \pm i s \\
\frac{1}{2}
\end{array}-r\right)}{\Gamma( \pm 2 i s)} F\left(\begin{array}{c} 
\pm i s \\
\frac{1}{2}
\end{array} ;-A\right) F\left(\begin{array}{c} 
\pm i s \\
\frac{1}{2}
\end{array} ;-B\right) d s
$$

equals

$$
\pi(1+A)^{\frac{1}{2}}(1+B)^{\frac{1}{2}} \frac{1+A+r+B}{(1+A+r+B)^{2}+4 r A B} .
$$

The denominator in (2.11) is positive.

Proof. Note first that

$$
(1+A+r+B)^{2}+4 r A B>(r+B)^{2}-4 r B=(r-B)^{2} \geq 0
$$

for $r, B \geq 0$ and $A>-1$, hence the last statement is true..

We may assume $B>0$, since the $B=0$ case is proved in Lemma 2.3.

By the third line of [2], p 999, 9.131.1 and by (ii) of Lemma 2.1 we have that

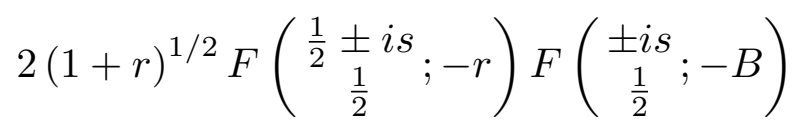

equals

$$
\sum_{\epsilon \in\{-1,1\}} F\left(\begin{array}{c}
-i s, i s \\
\frac{1}{2}
\end{array}-(\sqrt{r} \sqrt{B+1}+\epsilon \sqrt{B} \sqrt{r+1})^{2}\right) .
$$

Applying again the third line of [2], p 999, 9.131.1 and then using Lemma 2.3, taking into account (2.3) and (2.4) with $r$ and $B$ in place of $x$ and $y$, we get that (2.10) equals

$$
\frac{\pi(1+A)^{\frac{1}{2}}}{2(1+r)^{1 / 2}} \sum_{\epsilon \in\{-1,1\}} \frac{\sqrt{r+1} \sqrt{B+1}+\epsilon \sqrt{r} \sqrt{B}}{1+(\sqrt{r} \sqrt{B+1}+\epsilon \sqrt{B} \sqrt{r+1})^{2}+A} .
$$

Then by the identities

$$
(1+A+r+B+2 r B)^{2}-4 r(1+r) B(1+B)=(1+A+r+B)^{2}+4 r A B
$$

and

$$
\sum_{\epsilon \in\{-1,1\}}(\sqrt{r+1} \sqrt{B+1}+\epsilon \sqrt{r} \sqrt{B})\left(1+(\sqrt{r} \sqrt{B+1}-\epsilon \sqrt{B} \sqrt{r+1})^{2}+A\right)=
$$




$$
=2 \sqrt{r+1} \sqrt{B+1}(1+A+r+B)
$$

we get the lemma.

\section{Proof of the theorem}

We now turn to the proof of Theorem 1.1. Let $0<T<S<1$ be given from now on. We note that $A_{t}(S, T)$ is obviously an entire function of $t$, hence it is enough to prove the identity for real $t$.

LEMMA 3.1. Assume that there is an $r_{0}>0$ such that

$$
\frac{1}{\pi} \int_{0}^{\infty} \frac{\Gamma( \pm 2 i t) \Gamma^{2}\left(\frac{1}{2} \pm 2 i t\right) F\left(\begin{array}{c}
\frac{1}{2} \pm 2 i t \\
\frac{1}{2}
\end{array} ;-r\right)}{\Gamma( \pm 4 i t)}\left(A_{t}(S, T)-\frac{\pi}{\sqrt{1-T} \sqrt{1-S}}\right) d t=0
$$

for every $r>r_{0}$. Then

$$
A_{t}(S, T)=\frac{\pi}{\sqrt{1-T} \sqrt{1-S}}
$$

for every real $t$.

Proof. By Lemma 2.1 (i) for $0<Y<1$ one has that

$$
F\left(\begin{array}{c}
2 i t,-2 i t \\
\frac{1}{2}
\end{array} Y\right)=\cos (4 i t \arg (\sqrt{1-Y}+i \sqrt{Y}))
$$

where the argumentum of a complex number lying in the right half-plane is taken in $\left(-\frac{\pi}{2}, \frac{\pi}{2}\right)$. Estimating the first hypergeometric function in (1.1) by this relation and estimating the second one directly by $(2.2)$, we get that

$$
A_{t}(S, T)=O_{S, T}\left(e^{(2 \pi-c(S, T)) t}\right)
$$

with some constant $c(S, T)>0$ depending only on $S$ and $T$.

Using (3.2), (2.9) and the Stirling formula we see that the left-hand side of (3.1) is absolutely convergent for real $r$, moreover, this integral extends as a holomorphic function of $r$ to a domain in the complex plane containing the positive real line. Therefore by the unicity theorem we see that (3.1) is true for every $r>0$. Using (2.9) one then sees that the Fourier transform of the function

$$
\frac{\Gamma( \pm 2 i t) \Gamma^{2}\left(\frac{1}{2} \pm 2 i t\right)}{\Gamma( \pm 4 i t)}\left(A_{t}(S, T)-\frac{\pi}{\sqrt{1-T} \sqrt{1-S}}\right)
$$


is identically 0 . The lemma follows.

Hence for the proof of the theorem it is enough to show (3.1) for large enough $r$. The next two lemma will be useful to compute the left-hand side of (3.1).

LEMMA 3.2. For every $r>0$ one has that

$$
\frac{1}{\pi} \int_{0}^{\infty} \frac{\Gamma( \pm 2 i t) \Gamma^{2}\left(\frac{1}{2} \pm 2 i t\right) F\left(\begin{array}{c}
\frac{1}{2} \pm 2 i t \\
\frac{1}{2}
\end{array} ;-r\right)}{\Gamma( \pm 4 i t)} \frac{\pi}{\sqrt{1-T} \sqrt{1-S}} d t
$$

equals

$$
(1+r)^{-1} \frac{\pi^{2}}{\sqrt{1-T} \sqrt{1-S}}
$$

Proof. By the third line of [2], p 999, 9.131.1 and by Lemma 2.2 with $T=0$ and $A=r$, using the substitution $s=2 t$ we get the statement.

LEMMA 3.3. For $T \leq z \leq S$ let

$$
B_{t}(z, S, T):=F\left(\begin{array}{c}
2 i t,-2 i t \\
\frac{1}{2}
\end{array} \frac{(1+\sqrt{z})(\sqrt{z}-\sqrt{T})}{2(1-\sqrt{T}) \sqrt{z}}\right) F\left(\begin{array}{c}
i t,-i t \\
\frac{1}{2}
\end{array} ;-\frac{(S-z)(1-z)}{(1-\sqrt{S})^{2} z}\right)
$$

Then for every $r>0$ and for every $T \leq z \leq S$ we have that

$$
\frac{1}{\pi} \int_{0}^{\infty} \frac{\Gamma( \pm 2 i t) \Gamma^{2}\left(\frac{1}{2} \pm 2 i t\right) F\left(\begin{array}{c}
\frac{1}{2} \pm 2 i t \\
\frac{1}{2}
\end{array} ;-r\right) B_{t}(z, S, T)}{\Gamma( \pm 4 i t)} d t=I_{1}(z) I_{2}(r, z)
$$

with the abbreviations

$$
I_{1}(z):=\pi(1-\sqrt{z})(\sqrt{z}+\sqrt{T})^{\frac{1}{2}}(\sqrt{z}+\sqrt{S})^{\frac{1}{2}}(1-\sqrt{T})^{\frac{1}{2}}(1-\sqrt{S})^{\frac{1}{2}}
$$

and

$$
I_{2}(r, z):=\frac{\sqrt{T}+\sqrt{S}-2 \sqrt{S} \sqrt{T}+R \sqrt{z}+z(\sqrt{T}+\sqrt{S}-2)}{E(r)+F(r) z+G(r) z^{2}}
$$

with the notations (note that $R$ also depends on $r$, but for simplicity we do not denote it)

$$
\begin{gathered}
R:=2 r(1-\sqrt{T})(1-\sqrt{S}) \\
E(r):=(\sqrt{T}+\sqrt{S}-2 \sqrt{S} \sqrt{T})^{2}+2 R \sqrt{S} \sqrt{T}
\end{gathered}
$$




$$
\begin{gathered}
F(r):=2(\sqrt{T}+\sqrt{S}-2)(\sqrt{T}+\sqrt{S}-2 \sqrt{S} \sqrt{T})+2 R(1+\sqrt{S} \sqrt{T}-2 \sqrt{T}-2 \sqrt{S})+R^{2}, \\
G(r):=(\sqrt{T}+\sqrt{S}-2)^{2}+2 R .
\end{gathered}
$$

For $T \leq z \leq S$ we have

$$
E(r)+F(r) z+G(r) z^{2}>0 .
$$

Proof. By [2], p 999, 9.133 we have that

$$
F\left(i t,-i t ;-\frac{(S-z)(1-z)}{(1-\sqrt{S})^{2} z}\right)=F\left(2 i t,-2 i t ;-\frac{(1+\sqrt{z})(\sqrt{S}-\sqrt{z})}{2(1-\sqrt{S}) \sqrt{z}}\right)
$$

by the conditions $0<T \leq z \leq S<1$.

Applying Lemma 2.4 with the substitution $s=2 t$ and with

$$
A=A(z):=-\frac{(1+\sqrt{z})(\sqrt{z}-\sqrt{T})}{2(1-\sqrt{T}) \sqrt{z}}, \quad B=B(z):=\frac{(1+\sqrt{z})(\sqrt{S}-\sqrt{z})}{2(1-\sqrt{S}) \sqrt{z}}
$$

( $A>-1$ and $B \geq 0$ are satisfied by our conditions), using the easily proved identities (recall the notations given in the text of the lemma)

$$
\begin{gathered}
1+A(z)=\frac{(1-\sqrt{z})(\sqrt{z}+\sqrt{T})}{2(1-\sqrt{T}) \sqrt{z}}, \quad 1+B(z)=\frac{(1-\sqrt{z})(\sqrt{z}+\sqrt{S})}{2(1-\sqrt{S}) \sqrt{z}}, \\
1+A(z)+r+B(z)=\frac{\sqrt{T}+\sqrt{S}-2 \sqrt{S} \sqrt{T}+R \sqrt{z}+z(\sqrt{T}+\sqrt{S}-2)}{2(1-\sqrt{T})(1-\sqrt{S}) \sqrt{z}}, \\
(1+A(z)+r+B(z))^{2}+4 r A(z) B(z)=\frac{E(r)+F(r) z+G(r) z^{2}}{4 z(1-\sqrt{T})^{2}(1-\sqrt{S})^{2}}
\end{gathered}
$$

we obtain (3.3). The inequality (3.5) follows from (3.8) and the last statement of Lemma 2.4. The lemma is proved.

LEMMA 3.4. Assume that there is an $r_{0}>0$ such that

$$
Q_{S, T}(r):=(1+r) \int_{T}^{S} \frac{I_{2}(r, z)}{(1+\sqrt{z}) \sqrt{\sqrt{z}-\sqrt{T}} \sqrt{\sqrt{S}-\sqrt{z}}} d z
$$


equals

$$
\frac{\pi}{\sqrt{1-T} \sqrt{1-S} \sqrt{1-\sqrt{T}} \sqrt{1-\sqrt{S}}}
$$

for every $r>r_{0}$, where $I_{2}(r, z)$ is defined in Lemma 3.3. Then Theorem 1.1 is true.

Proof. This follows at once from Lemma 3.1, Lemma 3.2, (1.1) and Lemma 3.3.

LEMMA 3.5. We have that

$$
Q_{S, T}(r)-\frac{\pi}{\sqrt{1-T} \sqrt{1-S} \sqrt{1-\sqrt{T}} \sqrt{1-\sqrt{S}}}=O_{S, T}\left(\frac{1}{r}\right)
$$

as $r \rightarrow+\infty$.

Proof. By (3.4), (3.7), (3.8) we have the identity

$$
(1+r) I_{2}(r, z)=\frac{1}{2(1-\sqrt{T})(1-\sqrt{S}) \sqrt{z}} \frac{(1+r)(1+A(z)+r+B(z))}{(1+A(z)+r+B(z))^{2}+4 r A(z) B(z)},
$$

so $(1+r) I_{2}(r, z)$ equals

$$
\frac{1}{2(1-\sqrt{T})(1-\sqrt{S}) \sqrt{z}}+O_{S, T}\left(\frac{1}{r}\right)
$$

as $r \rightarrow+\infty$, uniformly for $T \leq z \leq S$. By (3.9) we see that for the proof of the present lemma it is enoiugh to show that

$$
\int_{T}^{S} \frac{1}{(1+\sqrt{z}) \sqrt{\sqrt{z}-\sqrt{T}} \sqrt{\sqrt{S}-\sqrt{z}}} \frac{d z}{2 \sqrt{z}}=\frac{\pi \sqrt{1-\sqrt{T}} \sqrt{1-\sqrt{S}}}{\sqrt{1-T} \sqrt{1-S}}
$$

By the substitution

$$
q=\frac{\sqrt{z}-\sqrt{T}}{\sqrt{S}-\sqrt{T}}
$$

we see that for (3.11) it is enough to show that

$$
\int_{0}^{1} \frac{1}{(1+\sqrt{T}+q(\sqrt{S}-\sqrt{T})) \sqrt{q} \sqrt{1-q}} d q=\frac{\pi \sqrt{1-\sqrt{T}} \sqrt{1-\sqrt{S}}}{\sqrt{1-T} \sqrt{1-S}} .
$$

Now, the left-hand side of (3.12) equals

$$
\frac{\pi}{(1+\sqrt{T})} F\left(\begin{array}{c}
\frac{1}{2}, 1 \\
1
\end{array} ;-\frac{\sqrt{S}-\sqrt{T}}{1+\sqrt{T}}\right)
$$


by [2], p 995, 9.111, and this shows (3.12) by the binomial theorem. The lemma follows.

LEMMA 3.6. For large enough positive $r$ we have that

$$
Q_{S, T}(r)-\frac{\pi}{\sqrt{1-T} \sqrt{1-S} \sqrt{1-\sqrt{T}} \sqrt{1-\sqrt{S}}}
$$

equals

$$
2 i n_{r} \pi \sqrt{(1-\sqrt{T})(1-\sqrt{S})} \frac{\sqrt{r}(1+r)}{\sqrt{F(r)^{2}-4 E(r) G(r)}},
$$

where $-4 \leq n_{r} \leq 4$ is an integer depending on $r$.

REMARK. The integer $n_{r}$ may depend also on $S$ and $T$, but $S$ and $T$ are fixed, so we do not denote it.

Proof. Since $F(r)^{2}-4 E(r) G(r)$ is a polynomial in $r$ of exact order 4 and $E(r)+F(r)+$ $G(r)$ is a polynomial in $r$ of exact order 2 we see (since obviously $E(r)>0$ and $G(r)>0$ ) that if $r$ is large enough, then the polynomial

$$
E(r)+F(r) z+G(r) z^{2}
$$

(we consider it as a polynomial in $z$ ) is of exact order 2, it does not have a double root in $z$ and $z=1$ is not a root, $z=0$ is not a root. One has

$$
E(r)+F(r) z+G(r) z^{2}=E(r)\left(1-\alpha_{1} z\right)\left(1-\alpha_{2} z\right)
$$

with some complex numbers $\alpha_{1}$ and $\alpha_{2}$ different from each other and from 1 and 0 . Note that $\alpha_{1}$ and $\alpha_{2}$ depend on $r$, but for simplicity we do not denote it.

Then it is easy to see (since writing $y=\sqrt{z}$ we have in (3.14) below the quotient of a polynomial of degree 3 and a polynomial of degree 5 in $y$, and the denominator has no double root, so we can take a partial quotient decomposition) that

$$
\sqrt{z} \frac{\sqrt{T}+\sqrt{S}-2 \sqrt{S} \sqrt{T}+R \sqrt{z}+z(\sqrt{T}+\sqrt{S}-2)}{(1+\sqrt{z})\left(E(r)+F(r) z+G(r) z^{2}\right)}
$$

equals

$$
\frac{a}{1+\sqrt{z}}+\frac{b}{1-\sqrt{\alpha_{1}} \sqrt{z}}+\frac{c}{1+\sqrt{\alpha_{1}} \sqrt{z}}+\frac{d}{1-\sqrt{\alpha_{2}} \sqrt{z}}+\frac{e}{1+\sqrt{\alpha_{2}} \sqrt{z}}
$$


for every $y=\sqrt{z}$ with some complex numbers $a, b, c, d$ and $e$ depending on $r$, where $\sqrt{\alpha_{1}}$ and $\sqrt{\alpha_{2}}$ are chosen arbitrarily, but they are fixed. Note that the denominators in (3.15) are nonzero for $T \leq z \leq S$ using (3.13) and (3.5).

By the substitution $q=\frac{\sqrt{z}-\sqrt{T}}{\sqrt{S}-\sqrt{T}}$ we see by (3.9), (3.4) (3.14) and (3.15) that $Q_{S, T}(r)$ equals

$$
2(1+r) \int_{0}^{1} \frac{\left(\frac{a}{1+\sqrt{z}}+\frac{b}{1-\sqrt{\alpha_{1}} \sqrt{z}}+\frac{c}{1+\sqrt{\alpha_{1}} \sqrt{z}}+\frac{d}{1-\sqrt{\alpha_{2}} \sqrt{z}}+\frac{e}{1+\sqrt{\alpha_{2}} \sqrt{z}}\right)}{\sqrt{q} \sqrt{1-q}} d q
$$

where (by a slight abuse of notation) $\sqrt{z}$ now denotes a function of $q$, namely

$$
\sqrt{z}:=\sqrt{T}+q(\sqrt{S}-\sqrt{T})
$$

Now, let $u$ be any such complex number for which

$$
1-u(\sqrt{T}+q(\sqrt{S}-\sqrt{T}))
$$

is nonzero for $0 \leq q \leq 1$. Then

$$
\int_{0}^{1} \frac{1}{\sqrt{q} \sqrt{1-q}} \frac{1}{(1-u(\sqrt{T}+q(\sqrt{S}-\sqrt{T})))} d q
$$

equals

$$
\frac{\pi}{1-u \sqrt{T}} F\left(\begin{array}{c}
\frac{1}{2}, 1 \\
1
\end{array} ; u \frac{\sqrt{S}-\sqrt{T}}{1-u \sqrt{T}}\right)=\frac{\Gamma^{2}\left(\frac{1}{2}\right)}{1-u \sqrt{T}}\left(\frac{1-u \sqrt{S}}{1-u \sqrt{T}}\right)^{-\frac{1}{2}},
$$

where we used also the binomial theorem. Hence $Q_{S, T}(r)$ equals the sum of

$$
\begin{gathered}
2(1+r) a \frac{\pi}{(1+\sqrt{T})}\left(\frac{1+\sqrt{S}}{1+\sqrt{T}}\right)^{-\frac{1}{2}}, \\
D_{1}:=2(1+r) b \frac{\pi}{\left(1-\sqrt{\alpha_{1}} \sqrt{T}\right)}\left(\frac{1-\sqrt{\alpha_{1}} \sqrt{S}}{1-\sqrt{\alpha_{1}} \sqrt{T}}\right)^{-\frac{1}{2}}, \\
D_{2}:=2(1+r) c \frac{\pi}{\left(1+\sqrt{\alpha_{1}} \sqrt{T}\right)}\left(\frac{1+\sqrt{\alpha_{1}} \sqrt{S}}{1+\sqrt{\alpha_{1}} \sqrt{T}}\right)^{-\frac{1}{2}},
\end{gathered}
$$




$$
\begin{aligned}
& D_{3}:=2(1+r) d \frac{\pi}{\left(1-\sqrt{\alpha_{2}} \sqrt{T}\right)}\left(\frac{1-\sqrt{\alpha_{2}} \sqrt{S}}{1-\sqrt{\alpha_{2}} \sqrt{T}}\right)^{-\frac{1}{2}}, \\
& D_{4}:=2(1+r) e \frac{\pi}{\left(1+\sqrt{\alpha_{2}} \sqrt{T}\right)}\left(\frac{1+\sqrt{\alpha_{2}} \sqrt{S}}{1+\sqrt{\alpha_{2}} \sqrt{T}}\right)^{-\frac{1}{2}} .
\end{aligned}
$$

Note that using (3.8) we see that if

$$
E(r)+F(r) z+G(r) z^{2}=0
$$

then

$$
(1+A(z)+r+B(z))^{2}+4 r A(z) B(z)=0,
$$

so

$$
A(z) B(z)=-\frac{(1+A(z)+r+B(z))^{2}}{4 r}
$$

hence by (3.6) we have

$$
(\sqrt{z}-\sqrt{T})(\sqrt{z}-\sqrt{S})=-\frac{z(1-\sqrt{T})(1-\sqrt{S})}{(1+\sqrt{z})^{2}} \frac{(1+A(z)+r+B(z))^{2}}{r}
$$

Let us determine now the coefficients $a, b, c, d, e$. For this sake we note that (3.7) implies that

$$
\sqrt{T}+\sqrt{S}-2 \sqrt{S} \sqrt{T}+R \sqrt{z}+z(\sqrt{T}+\sqrt{S}-2)
$$

equals

$$
2(1-\sqrt{T})(1-\sqrt{S}) \sqrt{z}(1+A(z)+r+B(z)),
$$

so by the equality of (3.14) and (3.15), using also (3.13) we get that

$$
\begin{aligned}
& a=-\frac{(\sqrt{T}+\sqrt{S}-2 \sqrt{S} \sqrt{T})-R+(\sqrt{T}+\sqrt{S}-2)}{E(r)+F(r)+G(r)}, \\
& b=\frac{(1-\sqrt{T})(1-\sqrt{S})\left(1+A\left(\frac{1}{\alpha_{1}}\right)+r+B\left(\frac{1}{\alpha_{1}}\right)\right)}{\left(\alpha_{1}-\alpha_{2}\right)\left(1+1 / \sqrt{\alpha_{1}}\right) E(r)}, \\
& c=\frac{(1-\sqrt{T})(1-\sqrt{S})\left(1+A\left(\frac{1}{\alpha_{1}}\right)+r+B\left(\frac{1}{\alpha_{1}}\right)\right)}{\left(\alpha_{1}-\alpha_{2}\right)\left(1-1 / \sqrt{\alpha_{1}}\right) E(r)},
\end{aligned}
$$




$$
\begin{aligned}
& d=\frac{(1-\sqrt{T})(1-\sqrt{S})\left(1+A\left(\frac{1}{\alpha_{2}}\right)+r+B\left(\frac{1}{\alpha_{2}}\right)\right)}{\left(\alpha_{2}-\alpha_{1}\right)\left(1+1 / \sqrt{\alpha_{2}}\right) E(r)}, \\
& e=\frac{(1-\sqrt{T})(1-\sqrt{S})\left(1+A\left(\frac{1}{\alpha_{2}}\right)+r+B\left(\frac{1}{\alpha_{2}}\right)\right)}{\left(\alpha_{2}-\alpha_{1}\right)\left(1-1 / \sqrt{\alpha_{2}}\right) E(r)} .
\end{aligned}
$$

By the definition of $R, E(r), F(r)$ and $G(r)$ in Lemma 3.3 we easily see that

$$
a=\frac{1}{2(1-\sqrt{T})(1-\sqrt{S})(1+r)},
$$

hence (3.16) equals (3.10). Consequently

$$
Q_{S, T}(r)-\frac{\pi}{\sqrt{1-T} \sqrt{1-S} \sqrt{1-\sqrt{T}} \sqrt{1-\sqrt{S}}}=D_{1}+D_{2}+D_{3}+D_{4}
$$

see (3.17), (3.18), (3.19) and (3.20).

We claim that

$$
D_{i}^{2}=-4 \pi^{2}(1-\sqrt{T})(1-\sqrt{S}) \frac{r(1+r)^{2}}{\left(\alpha_{1}-\alpha_{2}\right)^{2} E^{2}(r)}
$$

for every $1 \leq i \leq 4$. For $i=1$ this follows from (3.22) and from (3.21) with $\sqrt{z}=\frac{1}{\sqrt{\alpha_{1}}}$, for $i=2$ this follows from (3.23) and from (3.21) with $\sqrt{z}=-\frac{1}{\sqrt{\alpha_{1}}}$, for $i=3$ this follows from (3.24) and from (3.21) with $\sqrt{z}=\frac{1}{\sqrt{\alpha_{2}}}$, for $i=4$ this follows from (3.25) and from (3.21) with $\sqrt{z}=-\frac{1}{\sqrt{\alpha_{2}}}$.

We get from (3.27) for every $1 \leq i \leq 4$ that

$$
D_{i}=2 i \epsilon_{i} \pi \sqrt{(1-\sqrt{T})(1-\sqrt{S})} \frac{\sqrt{r}(1+r)}{\left(\alpha_{1}-\alpha_{2}\right) E(r)}
$$

where $\epsilon_{i}=1$ or $\epsilon_{i}=-1$ for every $i$. Since $\frac{1}{\alpha_{1}}$ and $\frac{1}{\alpha_{2}}$ are the two roots of $E(r)+F(r) z+$ $G(r) z^{2}=0$, so

$$
\alpha_{1} \alpha_{2}=\frac{G(r)}{E(r)}
$$

and

$$
\frac{1}{\alpha_{2}}-\frac{1}{\alpha_{1}}=\delta \frac{\sqrt{F^{2}(r)-4 E(r) G(r)}}{G(r)},
$$

where $\delta=1$ or $\delta=-1$. Then we get

$$
\left(\alpha_{1}-\alpha_{2}\right) E(r)=\delta \sqrt{F^{2}(r)-4 E(r) G(r)} .
$$


By (3.26), (3.28) and (3.29) we get the lemma.

LEMMA 3.7. If $r$ is a large enough positive number, then we have that

$$
Q_{S, T}(r)=\frac{\pi}{\sqrt{1-T} \sqrt{1-S} \sqrt{1-\sqrt{T}} \sqrt{1-\sqrt{S}}}
$$

Proof. It is enough to show that the integer $n_{r}$ in Lemma 3.6 is 0 for large enough $r$.

Since $F^{2}(r)-4 E(r) G(r)$ is a polynomial in $r$ of exact order 4 , therefore Lemma 3.6 implies that

$$
\left|Q_{S, T}(r)-\frac{\pi}{\sqrt{1-T} \sqrt{1-S} \sqrt{1-\sqrt{T}} \sqrt{1-\sqrt{S}}}\right| \gg_{S, T} \frac{\left|n_{r}\right|}{\sqrt{r}}
$$

for large $r$. By Lemma 3.5 this implies for large enough $r$ that $n_{r}=0$. The lemma is proved.

Using Lemmas 3.4 and 3.7 we see that the proof of the theorem is now complete.

ACKNOWLEDGEMENT. The author is grateful to the referee for giving useful advice and encouraging him to simplify significantly the first version of the paper.

\section{References}

[1] G.E. Andrews, R. Askey, R. Roy, Special Functions, Cambridge Univ. Press, 1999

[2] I.S. Gradshteyn, I.M. Ryzhik, Table of integrals, series and products, 6th edition, Academic Press, 2000

[3] L.J. Slater, Generalized hypergeometric functions, Cambridge Univ. Press, 1966 